\title{
Complex- $q$ zeros of the partition function of the Potts model with long-range interactions
}

\author{
Zvonko Glumac $^{1}$, \\ Faculty of Electrical Engineering, Kneza Trpimira 2B, 31000 Osijek, Croatia \\ Katarina Uzelac \\ Institute of Physics, P.O.B. 304, Bijenička 46, HR-10000 Zagreb, Croatia
}

\begin{abstract}
The zeros of the partition function of the ferromagnetic $q$-state Potts model with long-range interactions in the complex- $q$ plane are studied in the mean-field case, while preliminary numerical results are reported for the finite $1 d$ chains with powerlaw decaying interactions. In both cases, at any fixed temperature, the zeros lie on the arc-shaped contours, which cross the positive real axis at the value for which the given temperature is transition temperature. For finite number of spins the positive real axis is free of zeros, which approach to it in the thermodynamic limit. The convergence exponent of the zero closest to the positive real- $q$ axis is found to have the same value as the temperature critical exponent $1 / \nu$.
\end{abstract}

PACS: 05.50.+q, 64.60.Cn

Key words: Phase transitions, $q$-state Potts model, Complex- $q$ zeros, Long-range interactions

\section{Introduction}

Several decades ago, it was shown that the study of distribution of the partitionfunction zeros in the complex plane of an appropriate variable, can provide relevant informations on phase transitions. In their pioneering work, Lee and Yang $[1,2]$ have shown the connection between the existence of phase transition and the distribution of partition function zeros of the ferromagnetic

1 Corresponding author. tel.: (385)-031-208-900; fax: (385)-031-208-905; e-mail: zvonko@vrabac.ifs.hr. 
Ising model in the complex plane of a symmetry breaking field. Later on, $[3,4]$, similar connection was established for the partition function zeros in the complex-temperature plane. In both cases, the singularities at the transition point are closely related to the way that the zeros approach (either with temperature or with the size of the system) to the positive real axis.

The partition function zeros were investigated for number of models, including the Potts model both in complex-field $[5,6]$ and in complex-temperature [7-10] plane. In the Potts model a relevant quantity is also the number of states $q$, often considered as a continuous parameter, which is formally possible on the basis of its graphical representation [11] as it will be discussed later in text. This gives the motivation to investigate the zeros for this model also in the plane of complex $q$. Such an idea was already formulated $[12,13]$ but mostly in context of chromatic polynomials in connection with studies of the ground state of the anti-ferromagnetic Potts model [14-20]. Only a few works on complex- $q$ plane address the ferromagnetic Potts model at finite temperatures. The papers by Chang and Shrock, [21], study the loci of partition function zeros in $2 d$ Potts model with different boundary conditions. In their very recent paper Kim and Creswick [22] give a systematic study of both Fisher and complex- $q$ zeros in the $2 d$ Potts model with short-range interactions and find the similarity between the scaling property of complex- $q$ zeros and the den Nijs expression for the thermal critical exponent.

In the present paper we investigate the zeros in the complex- $q$ plane in connection with the first- and second-order phase transitions in the ferromagnetic Potts model. To this purpose we consider here the Potts model with long-range power-law decaying interactions, which already in one dimension displays diversity of critical regimes and embraces most of the nontrivial aspects of phase transitions, characteristic of the Potts model in general. The limiting case of this model, where all the interactions are equal (and which represents the mean-field case of the model) has the advantage to be exactly solvable for arbitrary $q$.

In the next section we describe briefly the starting Hamiltonian and its graphical representation. In subsequent sections we present the results for the meanfield (Section 3) and the power-law decaying interactions case (Section 4). 


\section{Hamiltonian}

The ferromagnetic Potts model with interactions of arbitrary range is described by the Hamiltonian

$$
H=-\sum_{i, j} J_{i, j} \cdot \delta\left(s_{i}, s_{j}\right)
$$

where $s_{i}$ denotes the Potts spin at site $i$ of a $d$-dimensional lattice, which can take $q$ values, and $J_{i, j} \geq 0$ denotes the ferromagnetic interaction between spins at sites $i$ and $j$.

An impressive amount of studies of this model in the case of nearest neighbour interactions (for a review see $\mathrm{Wu}$ [23]) reveal its very complex critical behaviour which varies with $q$ and comprises transitions of different orders and belonging to various universality classes.

The model with long-range interactions was studied less, and mostly in two special cases. The first is the case when all the interactions are equal and it represents the mean-field limit of the model. It can be approached by the saddle point approximation [24-27] and in the thermodynamic limit the exact expressions, in particular those for critical temperature and exponents may be derived for all values of $q$. Second is the case with power-law interactions decaying with distance as $1 / r^{d+\sigma}$. The type of phase transition there depends in addition on the parameter of range $\sigma$, which in certain aspects has similar effects as the change of dimensionality [28]. The phase transition is there present in one dimension [29-31] and by varying $\sigma$, series of different regimes are met, which makes this $1 d$ model a useful paradigm for most of important features of the model in general (including also the first-order phase transition). It was a subject of number of recent studies [31-39].

The graphical representation of the Potts model [11] holds for arbitrary range of interactions. The partition function for the Hamiltonian (1) may be expressed as

$$
Z_{N}=\sum_{\text {all } \mathcal{G}} \prod_{\substack{\text { active } \\ \text { links }}} v_{i, j} q^{n(\mathcal{G})},
$$

where the summation is taken over all possible graphs $\mathcal{G}$. Each graph represents one possible configuration of connections between the $N$ spins, called active links, each of which contributes with a factor $v_{i, j}=\exp \left(J_{i, j} / k_{B} T\right)-1$. $n(\mathcal{G})$ denotes the number of of disconnected parts of the graph called clusters. The product is taken over all the active links of a considered graph. 
Whatever the interactions are, the number of clusters that a single graph contains ranges from one cluster (low temperature limit, where all spins are interconnected), up to at most $N$ clusters (high temperature limit where no active links are present and each spin is a cluster of size one). Thus, by collecting all the graphs with the same number of clusters, the partition function becomes simply an $N$-th order polynomial in $q$

$$
Z_{N}=\sum_{n=1}^{N} a_{n}\left(T, J_{i, j}, N\right) q^{n}
$$

This made possible to treat the number of states $q$ as a continuous parameter. It also gives the possibility to examine the zeros of the partition function as the zeros of the $N$-th order polynomial in $q$.

The coefficients $a_{n}$ are functions of temperature, the finite size and interactions, and are quite complicated in the general case (1), but can be written in more compact form for the special cases considered here.

\section{The mean-field case}

The simplest case of long-range interactions is the one where all the interactions are set to be equal, $J_{i, j}=J$, which reduces the Hamiltonian (1) to the form

$$
\mathcal{H}_{M F}=H_{M F} / k_{B} T=-\frac{K}{N} \sum_{i=1}^{N-1} \sum_{j=1}^{N-i} \delta\left(s_{i}, s_{i+j}\right),
$$

where $K=J / k_{B} T$, and division by $N$ is necessary to keep the summation finite. This model corresponds to the mean-field limit. It was solved by the saddle point approximation [24]. The phase transition is there of the first order for $q>2$ and of the second order for $q \leq 2$. The exact expressions for the inverse of the transition temperature $K_{t}$ is given by

$$
K_{t}=2 \frac{q-1}{q-2} \ln (q-1)
$$

for $q>2$, and by $K_{c}=q$ for $q \leq 2$. Exact analytical expressions may be derived also for the critical exponents matching the values obtained in the mean-field regime of the short-range interaction model [27] (except those related to the correlation function, as it will be discussed later in text). 
The graph representation (2) reduces in the mean-field limit to

$$
Z_{N}=\sum_{\text {all } \mathcal{G}} v^{b(\mathcal{G})} q^{n(\mathcal{G})},
$$

where the active links have all the same strength $v=\exp (K / N)-1$, independently of distance between the spins they connect and $b(\mathcal{G})$ denotes the number of active links in a given graph $\mathcal{G}$. Written as a polynomial in $q$ the above equation is equal to

$$
Z_{N}=\sum_{n=1}^{N} a_{n}(v, N) q^{n},
$$

where the coefficients $a_{n}$ are real and positive and depend on temperature and size $N$ only. (Notice, that, since the number of active bonds may reach up to $N(N-1) / 2$, the above partition function could equally be written as a polynomial in temperature variable $v$, which would be of order $N(N-1) / 2$.)

\subsection{Numerical results}

For relatively small sizes the evaluation of coefficients and finding the zeros of the polynomial (7) in the complex plane may easily be performed numerically. Numerical results for sizes up to $N=35$ spins are presented in Figs. 1 (a)-(c) for the three different temperatures, $K=0.5,2,4.540457$. These temperatures are the transition temperatures of the mean-field model with $q=0.5,2$, and 8 respectively. Each figure displays the data obtained for several sizes.

One may observe that in all the three cases the zeros lie on contours similar but different from circle which cross the real axis near the value of $q$, for which the given temperature is the transition temperature. The zeros are absent from the positive real axis, but approach to it with increasing $N$.

We were thus interested to perform a more precise extrapolation of this crossing point in the thermodynamic limit and studied the convergence of the zero closest to the positive real axis with $N$. Further, we have examined the pos-

sible connection of the convergence of the closest zero with $N$ to the scaling properties of the model near criticality.

To this purpose we have used Burlisch and Stoer (BST) extrapolation procedure [40,41]. This algorithm was shown [41] to converge rapidly, needs a modest amount of data and, in advantage over the similar algorithm by van den Broeck and Schwartz (VBS) [42], it is less sensitive to the rounding errors. 
In short, BST algorithm extrapolates a sequence of the form

$$
A(N)=A_{\infty}+a_{1} \cdot N^{-\omega}+a_{2} \cdot N^{-2 \omega}+\cdots
$$

in the $N \rightarrow \infty$ limit by a set of transformations recursively given by

$$
\begin{aligned}
A_{m}^{(N)} & =A_{m-1}^{(N+1)} \\
& +\left(A_{m-1}^{(N+1)}-A_{m-1}^{(N)}\right) \cdot\left[\left(\frac{N+m}{N}\right)^{\omega}\left(1-\frac{A_{m-1}^{(N+1)}-A_{m-1}^{(N)}}{A_{m-1}^{(N+1)}-A_{m-2}^{(N+1)}}\right)-1\right]^{-1},
\end{aligned}
$$

where $A_{-1}^{(N)}=0$ and $A_{0}^{(N)}=q_{1,2}^{(0)}(N)$ are the input data and $\omega$ is a free parameter.

If the original series is of the form:

$$
A(N)=A_{\infty}+c_{1} \cdot N^{-\omega_{1}}+c_{2} \cdot N^{-\omega_{2}}+\cdots
$$

it incorporates the leading convergence exponent as $\omega$, while the higher order approximants converge as $N^{-\omega^{\prime}}$, where $\omega^{\prime}=\min \left(2 \omega_{1}, \omega_{2}\right)$. Although it was shown, that the extrapolations are insensitive to the choice of $\omega$ in a wide range of values, it influences its convergence speed. Thus, by maximising the convergence of higher approximants one can in the same time obtain both the extrapolated value and the leading convergence exponent. Also, when the extrapolated value is known in advance, $\omega$ yields the leading convergence exponent.

We have examined the convergence of the real and imaginary parts of the zero closest to the real axis, denoted as $q_{1,2}^{(0)}(N)$ for several temperatures using the numerical results ranging from $N=21$ to $N=35$.

In the extrapolation of both real and imaginary part $q_{1,2}^{(0)}(N)$, the same parameter $\omega$ was used and was fixed by the constraint $q_{2}^{(0)}(N \rightarrow \infty) \rightarrow 0$.

The results of extrapolations are presented on Table 1 . By $Q_{0}$ is denoted the value of $q$ for which the considered temperature is known to be the transition temperature. All the data were obtained by requiring that condition $q_{2}^{(0)}(N) \rightarrow 0$ should be fulfilled with precision of $10^{-6}$.

The presented results indicate that:

(i) loci of complex- $q$ zeros intersect the positive real- $q$ axis at value $q_{1}=Q_{0}$. The values agree up to a few percents. 
Table 1

The BST extrapolation results of the positions of closest zeros.

\begin{tabular}{ccccccc}
\hline$K$ & 0.1 & 0.5 & 1.0 & 2.0 & 2.772589 & 4.540457 \\
$Q_{0}$ & 0.1 & 0.5 & 1.0 & 2.0 & 3.0 & 8.0 \\
$q_{1}$ & 0.09998 & 0.504 & 0.95 & 2.05 & 3.02 & 8.00009 \\
$\omega$ & 0.34 & 0.33 & 0.33 & 0.55 & 0.91 & 1.19 \\
\hline
\end{tabular}

(ii) Power-law convergence of the closest zero is observed in all the cases considered.

The convergence exponent $\omega$ represents the scaling with size of the distance from the real axis to the closest zero. Before proceeding with the finite-size scaling analysis it is important to notice that here the scaling is not done with the linear size, but rather with volume, more precisely, with number of spins, since in the infinitely coordinated model the distance as well as the dimensionality lose their meaning. In this respect the considered mean-field limit differs from the standard mean-field approach to the short-range interaction model. As pointed out by Botet et al. $[43,44]$ the basic scaling quantity in the infinitely coordinated system is not the correlation length, but the "correlation number" $N_{c}$, which scales with temperature with the critical exponent $\nu^{*}=\nu_{M F} \cdot d_{c}$. The exponent $\nu_{M F}$ and $d_{c}$ are, respectively, the mean-field value of the critical exponent $\nu$ and the upper critical dimension (the dimension above which the mean-field regime sets on) of the short-range model.

Thus, the temperature critical exponent for the present model, where only the scaling with the number of spins can be applied will be $1 / \nu^{*}$ and not $1 / \nu_{M F}$. In the Potts model $\nu_{M F}=1 / 2$ both for $q<2$ and $q=2$, while $d_{c}=4$ for $q=2$, but $d_{c}=6$ for $q<2$ for symmetry reasons. This gives $\nu^{*}=2$ for $q=2$ and $\nu^{*}=3$ for $q<2$.

The results presented in Table 1 clearly show that in the regime of secondorder phase transition $(q \leq 2)$ the convergence exponent coincides up to a few percents with the temperature critical exponent $1 / \nu^{*}$. This result should be compared to the one in complex temperature plane, where it was shown [45] that the distance of the zero closest to the real axis scales as the temperature critical exponent. Similar behaviour in a complex- $q$ plane was recently observed in the $2 d$ ferromagnetic Potts model by Kim and Creswick [22], but the precision of their results did not permit them to be conclusive whether the obtained convergence exponent was indeed equal to the exponent $1 / \nu$.

In cases $q=3$ and $q=8$ we obtain approximately linear convergence, propor- 
tional to the system size, which is the form of scaling that is expected for the first-order transition.

\subsection{Large $N$ expansion}

In the limit $N \gg 1$ some analytical results may easily be derived by extending the mean-field solution by Kihara et al. For the large number of spins $N$, the free energy density, without the terms which are constant in the order parameter $S$, is given by

$$
\begin{aligned}
& \mathcal{F}(K, q, S)= \\
& -S^{2} \frac{K}{2} \frac{q-1}{q}+\frac{1+(q-1) S}{q} \ln [1+(q-1) S]+(q-1) \frac{1-S}{q} \ln (1-S)
\end{aligned}
$$

The partition function is given by

$$
Z_{N}(K, q) \sim \int_{0}^{1} \exp (-N \mathcal{F}) d S
$$

where the proportionality sign stands for all the omitted constant terms.

We limit our considerations here to the first-order regime of temperatures, where the free energy density at the transition shows two equal minima, and the partition function integral can be approximately solved by a saddle point method

$$
Z_{N} \sim 1+\exp \left[-N \mathcal{F}\left(S_{\min }\right)\right]
$$

The first term on the right hand side denotes the minimum at $S=0$, and the second term is the contribution of the second minimum at $S=S_{\min }>0$, which is obtained from the equation for the extremum

$$
K S=\ln \frac{1+(q-1) S}{1-S} .
$$

The straightforward way to find the complex- $q$ zeros would consist of two steps: first, by using (14) one should represent $\mathcal{F}$ as a function of $K$ and $q$ only; second, one should solve the equation $Z_{N}=0$ by analytic continuation of $q$ to the complex plane. Since $\mathcal{F}=\mathcal{F}_{R}+i \mathcal{F}_{I}$ then becomes complex, the vanishing of $Z_{N}$, as given by (13), requires

$$
\mathcal{F}_{R}=0, \quad N \mathcal{F}_{I}= \pm(2 m+1) \pi, \quad m=0,1, \ldots
$$


These two equations determine the position of complex conjugate pairs of zeros $\left(q_{1}^{(m)}, \pm q_{2}^{(m)}\right)$ in the complex- $q$ plane.

The first of the above mentioned steps cannot be performed in the straightforward way. Instead, we will solve (14) in variable $q$

$$
q=\frac{1-S}{S}[\exp (K S)-1]
$$

and represent $\mathcal{F}$ as function of $S$ and $K$.

$$
\mathcal{F}(K, S)=\frac{K}{2} \frac{S^{2}}{1-S} \frac{(1-S) \exp (K S)+1}{\exp (K S)-1}+\ln (1-S) .
$$

The equations (15) are then solved in the complex- $S$ plane and, by equation (16), mapped back into the complex- $q$ plane.

The solutions $q_{1,2}^{(m)}$ should comply with both equations (15). Since the first of equations (15) is $N$-independent, it contains all the possible positions of zeros for arbitrary $N$ including the limit $N \rightarrow \infty$. The zeros of the system of particular size $N$ are then determined as intersections of this curve with solutions of the second equation in (15). For large $N$ these intersections appear to be equally distributed along the the curve $\mathcal{F}_{R}=0$, so that it can be drawn as the contour containing zeros. (see Fig. 2 as an illustration). The curve crosses the positive real- $q$ axis at a point which is transition point for a given temperature.

Since the locus of zeros has a shape similar to a circle, we draw the circular contour (long dashed line) in order to stress the difference. The parameters of this circle are determined by the facts, that its center lies on the real- $q$ axis (since zeros appear in complex conjugate pairs only) and that it intersects the positive real- $q$ axis at $q$ solution of (5) and the negative real- $q$ axis at $q=-|q|$, solution of

$$
K=2 \frac{|q|+1}{|q|+2} \ln (|q|+1) .
$$

We were not able to give analytical expressions for all the zeros of the model, but the location of zeros closest to the positive real $S$ or $q$ axis is a relatively easy task. 
Since we limit here to the first-order transitions, the temperature will be fixed through the parameter $Q_{0}>2$

$$
K=2 \frac{Q_{0}-1}{Q_{0}-2} \ln \left(Q_{0}-1\right)
$$

In the thermodynamic limit the second minimum of $\mathcal{F}(S)$ appears at $S_{0}=$ $\left(Q_{0}-2\right) /\left(Q_{0}-1\right)$. For large but finite $N$, we will expand free energy (17) around the minimum, $S=S_{0}+s$. The small $s \ll S_{0}$ vanishes as $N \rightarrow \infty$, so we expect to have, to leading order in $1 / N$,

$$
s=s_{1}+i s_{2}, \quad s_{1}=\frac{a}{N^{x_{1}}}, \quad s_{2}=\frac{b}{N^{x_{2}}},
$$

for real constants $a, b, x_{1}, x_{2}$. The expansion of (17) and the constraint (15) give the equation

$$
\begin{aligned}
& \mathcal{F}=\left(Q_{0}-1\right)\left[1-\ln \left(Q_{0}-1\right)\left(A_{1}-B_{1}\right)\right] s+\mathcal{O}\left(s^{2}\right)=0 \pm i \frac{2 m+1}{N} \pi \\
& A_{1}=\left(\frac{K-Q_{0}+1}{Q_{0}}+\frac{2}{Q_{0}-2}\right), B_{1}=\frac{Q_{0}-1}{Q_{0}\left(Q_{0}-2\right)} K-1
\end{aligned}
$$

The closest zero has the smallest imaginary part, so its index is $m=0$. By inserting (20) in the above equation, one reads

$$
a=0, \quad b=\frac{\pi}{\left(Q_{0}-1\right)-\ln \left(Q_{0}-1\right)\left(A_{1}-B_{1}\right)}, \quad x_{2}=1,
$$

while $x_{1}$ is not defined. Mapping back to the complex- $q$ plane by using the equation (16) gives

$$
\begin{aligned}
q_{1}^{(0)}(N) & =Q_{0}+\frac{\left(Q_{0}-1\right)^{2}}{Q_{0}-2}\left(K-Q_{0}\right) \frac{a}{N^{x_{1}}} \\
& +\frac{\left(Q_{0}-1\right)^{2}}{Q_{0}-2}\left\{\frac{K}{2}\left[K-2\left(Q_{0}-1\right)\right]+\frac{Q_{0}-1}{Q_{0}-2}\left(Q_{0}-K\right)\right\}\left(\frac{a^{2}}{N^{2 x_{1}}}-\frac{b^{2}}{N^{2 x_{2}}}\right) \\
& +\mathcal{O}\left(N^{-3 x}\right), \\
q_{2}^{(0)}(N) & =\frac{\left(Q_{0}-1\right)^{2}}{Q_{0}-2}\left(K-Q_{0}\right) \frac{b}{N^{x_{2}}} \\
& +\frac{\left(Q_{0}-1\right)^{2}}{Q_{0}-2}\left\{\frac{K}{2}\left[K-2\left(Q_{0}-1\right)\right]+\frac{Q_{0}-1}{Q_{0}-2}\left(Q_{0}-K\right)\right\} \frac{2 a b}{N^{x_{1}+x_{2}}} \\
& +\mathcal{O}\left(N^{-3 x}\right) .
\end{aligned}
$$


Finally, by inserting values for $a, b, x_{2}$ from (22), one obtains the large- $N$ dependence of the distance of the closest zero from the real axis in the case of the first-order transitions.

$$
\begin{aligned}
q_{1}^{(0)}(N) & =Q_{0}-\frac{\left(Q_{0}-1\right)^{2}}{Q_{0}-2}\left\{\frac{K}{2}\left[K-2\left(Q_{0}-1\right)\right]+\frac{Q_{0}-1}{Q_{0}-2}\left(Q_{0}-K\right)\right\} \frac{b^{2}}{N^{2}} \\
& +\mathcal{O}\left(N^{-3 x}\right), \\
q_{2}^{(0)}(N) & =\frac{\left(Q_{0}-1\right)^{2}}{Q_{0}-2}\left(K-Q_{0}\right) \frac{b}{N}+\mathcal{O}\left(N^{-3 x}\right),
\end{aligned}
$$

The above expressions can be checked numerically. For illustration, the numerical data of the closest zeros, for the temperature critical for $q=8$ are presented in Table 2 for different sizes $N$.

Table 2

The location of the closest zeros for the temperature given by the relation (19) for $Q_{0}=8 . N$ denotes the number of the system considered.

\begin{tabular}{ccc}
\hline$N$ & $q_{1}^{(m=0)}(N)$ & $q_{2}^{(m=0)}(N)$ \\
5000 & 7.99999543669504 & $-8.679091351481774 \cdot 10^{-3}$ \\
6000 & 7.99999683103614 & $-7.232577480179549 \cdot 10^{-3}$ \\
7000 & 7.99999767178073 & $-6.199352825621011 \cdot 10^{-3}$ \\
8000 & 7.99999821745666 & $-5.424434119811201 \cdot 10^{-3}$ \\
9000 & 7.99999859157044 & $-4.821719459791061 \cdot 10^{-3}$ \\
10000 & 7.99999885917194 & $-4.339547669719317 \cdot 10^{-3}$ \\
11000 & 7.99999905716679 & $-3.945043440975050 \cdot 10^{-3}$ \\
12000 & 7.99999920775815 & $-3.616289894012384 \cdot 10^{-3}$ \\
13000 & 7.99999932495365 & $-3.338113800831173 \cdot 10^{-3}$ \\
14000 & 7.99999941794470 & $-3.099677139486969 \cdot 10^{-3}$ \\
15000 & 7.99999949296515 & $-2.893032025972416 \cdot 10^{-3}$ \\
\hline
\end{tabular}

The data $q_{1,2}^{(0)}(N)$ from Table 2 and analogous series of data for different temperatures corresponding to $q=3$ and $q=2.1$ were extrapolated to the thermodynamic limit by using the BST algorithm.

The extrapolation parameter $\omega$ was fixed by the constraint $q_{2}^{(0)}(N) \rightarrow 0$. After we have made the extrapolations of $q_{1,2}^{(0)}(N)$ and found the values of $q_{1}(\infty)$ and $\omega$, we have performed alternative calculation of convergence exponents of 
$q_{1,2}^{(0)}(N)$. By assuming the convergence of the $q_{1,2}^{(0)}(N)$ to be of the form

$$
q_{1}^{(0)}(N)-q_{1}(\infty)=C_{1} \cdot N^{-\alpha_{1}}+\cdots, \quad q_{2}^{(0)}(N)=C_{2} \cdot N^{-\alpha_{2}}+\cdots,(25
$$

the convergence exponents $\alpha_{1,2}(N)$ for real and imaginary part of the closest zeros, can be expressed as

$$
\begin{aligned}
& \alpha_{1}(N)=-\frac{\ln \left\{\left[q_{1}^{(0)}(N)-q_{1}(\infty)\right] /\left[q_{1}^{(0)}(N+1)-q_{1}(\infty)\right]\right\}}{\ln [N /(N+1)]}, \\
& \alpha_{2}(N)=-\frac{\ln \left[q_{2}^{(0)}(N) / q_{2}^{(0)}(N+1)\right]}{\ln [N /(N+1)]}
\end{aligned}
$$

The above data for $\alpha_{1,2}(N)$ are extrapolated again by the BST algorithm. The complete set of extrapolated results is summarised in the Table 3.

Table 3

The results of the BST extrapolations performed on the closest zeros, $q_{1,2}(\infty)$, followed by parameter $\omega$ and convergence exponents $\alpha_{1,2}$, for set of temperatures determined by $Q_{0}$ and (19). The predicted convergence exponents $x_{1,2}$ as given by (24) are in the last two columns.

\begin{tabular}{cccccccc}
\hline$Q_{0}$ & $q_{1}(\infty)$ & $q_{2}(\infty)$ & $\omega$ & $\alpha_{1}$ & $\alpha_{2}$ & $x_{1}$ & $x_{2}$ \\
8 & 8.0000000000001 & $3 \cdot 10^{-13}$ & 1.0000 & 1.9999988 & 1.000000002 & 2 & 1 \\
3 & 3.000000000005 & $4 \cdot 10^{-12}$ & 1.00000 & 2.00003 & 0.99999997 & 2 & 1 \\
2.1 & 2.1002 & $9 \cdot 10^{-6}$ & 0.98 & 1.85 & 0.95 & 2 & 1 \\
\hline
\end{tabular}

We observe a full agreement between the analytically given values for $q$ and convergence exponents on one side, and the numerically calculated and extrapolated values for $q$ and exponents on the other side. Although the scaling in the case of the first-order phase transition is trivial and linearly proportional to system size, the results can be used to check the convergence of numerical results. For $q=3$ and $q=8$ the convergence exponent has improved compared to the earlier numerical results given in Table 1, confirming that larger error in this case comes from the small size of considered systems and that scaling is indeed linear with size as expected for the first-order phase transition. The loss of precision in extrapolations persists only for the case corresponding to $q=2.1$ and should be attributed to the crossover effects due to vicinity of the border between the first- and second-order transitions. 
For the partition function zeros lying on the curve in the appropriate complex plane, the normalised density of zeros $g$, is defined as [2]

$$
g=\frac{1}{N} \frac{d n}{d l}, \quad \int g d l=1
$$

where $d n$ is the number of zeros inside the arc of the curve of the length $d l$. In the case of the field- or temperature-driven second-order transitions, the density of zeros vanishes at the transition with an exponent connected to critical exponents [46]. On the contrary, at the first-order transition point, the density of zeros remains constant. To the end of this paragraph, we will examine the behaviour of density of complex- $q$ zeros in the vicinity of firstorder transition point. To this purpose, we notice that the large- $N$ expansion of $q_{1,2}(N)$ for the closest zeros (those with $m=0$ ) presented earlier in text, may be directly generalised to other values of $m$ as long as the condition $(2 m+1) \ll N$ is fulfilled. This leads to

$$
\begin{aligned}
q_{1}^{(m)}(N)-q_{1}(\infty) & =C_{1} \cdot\left(\frac{2 m+1}{N}\right)^{\alpha_{1}}+\cdots, \\
q_{2}^{(m)}(N) & =C_{2} \cdot\left(\frac{2 m+1}{N}\right)^{\alpha_{2}}+\cdots,
\end{aligned}
$$

with the constants $q_{1}(\infty), C_{1}$ and $C_{2}$ given by the relations $(24)$, where $1 / N$ is changed by $(2 m+1) / N$.

To calculate numerically density of zeros (27), we choose two neighbouring zeros, with $d l$ denoting geometrical distance between them,

$d l=\left\{\left[q_{1}^{(m+1)}-q_{1}^{(m)}\right]^{2}+\left[q_{2}^{(m+1)}-q_{2}^{(m)}\right]^{2}\right\}^{1 / 2}$. In that case density of zeros near positive real- $q$ axis becomes

$$
\begin{aligned}
g(m)= & \frac{1}{N}\left\{C_{1}^{2}\left(\frac{2 m+1}{N}\right)^{2 \alpha_{1}}\left[\left(1+\frac{2}{2 m+1}\right)^{\alpha_{1}}-1\right]^{2}\right. \\
& \left.+C_{2}^{2}\left(\frac{2 m+1}{N}\right)^{2 \alpha_{2}}\left[\left(1+\frac{2}{2 m+1}\right)^{\alpha_{2}}-1\right]^{2}\right\}^{-1 / 2}
\end{aligned}
$$

The edge $(m=0)$ density of zeros $g_{0}$, tends to constant value in the thermodynamic limit.

$$
g_{0}=\frac{1}{2 C_{2}}\left[1-8 \frac{C_{1}^{2}}{C_{2}^{2}} \frac{1}{N^{2}}+\cdots\right] \rightarrow \frac{1}{2 C_{2}}
$$


For example, for $Q_{0}=8$ and $K\left(Q_{0}=8\right)=4.540457014462$ this constant is

$$
\frac{1}{2 C_{2}}=\left[2 \frac{\left(Q_{0}-1\right)^{2}}{Q_{0}-2}\left(K-Q_{0}\right) b\right]^{-1}=0.011521939 \cdots
$$

For illustration, we check this result numerically by calculating for the values of $Q_{0}$ and $K$ from above, and $N=10000$, the twenty zeros closest to the positive real- $q$ axis and corresponding densities. They are presented in Table 4 .

The limiting value of the density at the transition point, is extracted by the BST extrapolation procedure (8) simply by changing variable $1 / N$ into $(2 m+$ 1) $/ N$.

$$
g_{m}=g_{0}+\tilde{g}\left(\frac{2 m+1}{N}\right)^{\omega}+\cdots
$$

The extrapolations give $g_{0}=1.152194 \cdot 10^{-2}$ for a wide range of $0.3<\omega<3.0$, which reproduces the analytically obtained value (31) in six significant digits.

We can conclude that the density of the partition function zeros in the complex$q$ plane behave in similar way as the density of zeros in the complex-temperature [3] or complex-field [46] planes: in thermodynamic limit it has constant value at the first-order transition point.

\section{The power-law decaying interactions}

The second case that we have considered is the ferromagnetic Potts model in one dimension with power-law decaying interactions. When taking the periodic boundary conditions, it is described by the reduced Hamiltonian

$$
\mathcal{H}_{P L}=-\frac{H_{P L}}{k_{B} T}=K \sum_{i=1}^{N-1} \sum_{j=1}^{N-i} \delta\left(s_{i}, s_{i+j}\right)\left[\frac{1}{j^{1+\sigma}}+\frac{1}{(N-j)^{1+\sigma}}\right] .
$$

Although in one dimension, the model is nontrivial and has a phase transition at nonzero temperature for all $q$ when $0<\sigma \leq 1$ [29-31]. This transition is of the mean-field type for low enough values of $\sigma, \sigma<\sigma_{c}(q)$, and is a non-trivial second-order phase transition for $\sigma>\sigma_{c}(q)$. In the classical regime $\sigma<\sigma_{c}(q)$, the transition is of the first order when $q>2[34,35,37]$. The exact position of the line separating the two regimes, $\sigma_{c}(q)$, is a difficult and still open question $[35,37,47]$. 
Table 4

The twenty zeros closest to the positive real- $q$ axis and the corresponding densities are shown at temperature determined from the relation (19) and $Q_{0}=8$ and for the $N=10000$ spins.

\begin{tabular}{cccc}
\hline$m$ & $q_{1}^{(m)}$ & $q_{2}^{(m)}$ & $g(m)$ \\
0 & 7.99999885917192 & -0.004339547669719 & 0.0115219529910602 \\
1 & 7.99998973259138 & -0.013018627057442 & 0.0115219974088187 \\
2 & 7.99997147966529 & -0.021697658591626 & 0.0115220714335465 \\
3 & 7.99994410086352 & -0.030376610375500 & 0.0115221750581617 \\
4 & 7.99990759689083 & -0.039055450520507 & 0.0115223082726076 \\
5 & 7.99986196868649 & -0.047734147149695 & 0.0115224710640261 \\
6 & 7.99980721742419 & -0.056412668400979 & 0.0115226634165474 \\
7 & 7.99974334451167 & -0.065090982430549 & 0.0115228853117394 \\
8 & 7.99967035159032 & -0.073769057415945 & 0.0115231367280630 \\
9 & 7.99958824053479 & -0.082446861559540 & 0.0115234176412815 \\
10 & 7.99949701345249 & -0.091124363091702 & 0.0115237280243068 \\
11 & 7.99939667268302 & -0.099801530274076 & 0.0115240678473335 \\
12 & 7.99928722079755 & -0.108478331402754 & 0.0115244370775645 \\
13 & 7.99916866059815 & -0.117154734811648 & 0.0115248356796385 \\
14 & 7.99904099511710 & -0.125830708875537 & 0.0115252636152771 \\
15 & 7.99890422761603 & -0.134506222013369 & 0.0115257208435568 \\
16 & 7.99875836158505 & -0.143181242691353 & 0.0115262073207999 \\
17 & 7.99860340074195 & -0.151855739426127 & 0.0115267230005962 \\
18 & 7.99843934903115 & -0.160529680787899 & 0.0115272678338162 \\
19 & 7.99826621062264 & -0.169203035403569 & 0.0115278417687404 \\
\hline & & & \\
\hline
\end{tabular}

In the graph representation, the difference from the mean-field case is that the contribution of active links depends on distance between the spins they connect. The contribution of active bonds may then have $N / 2$ (for $N$ even) or $(N-1) / 2$ (for $N$ odd) distinct values given by $v_{j}=\exp \left[K / j^{1+\sigma}+K /(N-\right.$ $\left.j)^{1+\sigma}\right]-1$. If we denote by $b_{j}(\mathcal{G})$ the number of active links of the length $j$ or $N-j$ in a graph $\mathcal{G}$, the partition function becomes

$$
Z_{N}=\sum_{\text {all } \mathcal{G}} v_{1}^{b_{1}(\mathcal{G})} v_{2}^{b_{2}(\mathcal{G})} \cdots q^{n(\mathcal{G})}
$$


or, in form of polynomial in $q$,

$$
Z_{N}=\sum_{n=1}^{N} a_{n}\left(v_{j}, N\right) q^{n} .
$$

The real and positive coefficients $a_{n}$ depend on temperature and $N$, but also on the parameter of range $\sigma$. This makes the calculations more demanding for the computer memory.

We have performed exact numerical calculations on chains with $N \leq 9$ spins for two sets of parameters, $(\sigma=0.3, K=0.576)$ and $(\sigma=0.8 K=0.8230)$. As in the previous case, the chosen temperatures are the critical temperatures known for certain values of $q$. They correspond to $q=5$ and $q=2$ respectively. Remark, however, that in the present case the critical temperatures are not known exactly. The values used here were obtained by the finite-range scaling approach [31]. The first and second set correspond respectively to the to the first- and second-order phase transition.

The numerical results for the loci of complex- $q$ zeros for sizes $N=6, \cdots, 9$ are presented in Figures 3 and 4 . In both examples are obtained the zeros lying on the arc-shaped curves, while the positive real axis is free of zeros. The zeros closest to it approach to it with increasing $N$.

As in the mean-field case, we have examined the convergence of the closest zeros to the thermodynamic limit by the BST algorithm, assuming the powerlaw form. The analysis included the data obtained for chains with $N=5$ to 9. The parameter $\omega$ was determined, as earlier, by requiring that $q_{2} \rightarrow 0$ in the thermodynamic limit. The results are presented in Table 5.

In the case of second order phase transition the expected crossing point with the real axis is obtained with precision better than $1 \%$. The leading convergence exponent given by $\omega$, is equal to 0.41 , which is close to the value for the temperature critical exponent $1 / \nu=0.48$ obtained earlier by finite-range scaling for $q=2$ and $\sigma=0.8[31]$.

In the case of the first-order transition the fit is more difficult. The discrepancy between the obtained crossing point and the value expected for the considered temperature is larger, about $20 \%$, while the convergence exponent, which in context of finite-size scaling should be linear for a first-order phase transition, differs by about $11 \%$. Larger error in determination of the value of $q$, may be attributed to both lower precision of the data for critical temperature obtained by finite-range scaling $[31,35]$ when the values of $\sigma$ are low and to the proximity of the second-order transition regime in the $(q, \sigma)$ plane, which might induce crossover effects for small sizes. 
Table 5

The loci of closest zeros and its BST extrapolation at $(K=0.576, \sigma=0.3)$ and $(K=0.823, \sigma=0.8)$

\begin{tabular}{ccccc}
\hline & $K=0.576$ & $\sigma=0.3$ & \\
\hline$N$ & $q_{1}^{(0)}(N)$ & $q_{2}^{(0)}(N)$ & $\omega$ \\
5 & -0.447413829093298 & 2.78806303559461 & \\
6 & 0.0734103695976816 & 2.88316232503579 & \\
7 & 0.486108948338677 & 2.88942370075814 & \\
8 & 0.818954244961081 & 2.85269647435491 & \\
9 & 1.09269817849075 & 2.79489503015230 & \\
$\infty$ & 4.2 & $3 \cdot 10^{-3}$ & 0.89 \\
\hline & $K=0.823$ & $\sigma=0.8$ & \\
\hline$N$ & $q_{1}^{(0)}(N)$ & $q_{2}^{(0)}(N)$ & $\omega$ \\
5 & -0.418572384698087 & 3.69577294340190 & \\
6 & 0.179124021423254 & 3.55212427658784 & \\
7 & 0.581821465484680 & 3.37723067427777 & \\
8 & 0.866098195024489 & 3.20669717997192 & \\
9 & 1.074705295884560 & 3.05042285906340 & \\
$\infty$ & 2.01 & $3 \cdot 10^{-3}$ & 0.41 \\
\hline
\end{tabular}

Since in these preliminary calculations we dispose with small number of data, we have not calculated the density of zeros in the case of power-law model.

\section{Conclusion}

We have studied the complex- $q$ zeros of the partition function of the ferromagnetic Potts model with long-range interactions in context of the firstand second-order phase transitions in this model. Two cases are presented: the mean-field limit and a few examples of the case with power-law decaying interactions.

We conclude that the positions of the zeros may be analysed in a similar way as the zeros in the plane of complex field or temperature and that they can give useful informations on the phase transition in ferromagnetic Potts models.

Numerical results for relatively small systems show the similar picture as in the case of zeros in the complex field or temperature plane. In all the cases 
considered the zeros lie on smooth curves that intersect the real positive axis at value for which the given temperature is the transition temperature. For finite systems the positive real axis is free of zeros, but they approach to it with increasing size.

The distance of the closest zero from the real axis is found to scale with size as the temperature critical exponent $1 / \nu$. In the mean-field case, this result could be obtained with a good precision for series of different temperatures corresponding to different transition regimes. In the case of power-law interactions, the preliminary results obtained with less precision suggest the same behaviour. This result is similar to behaviour in the complex temperature plane, and it supports recent findings by Kim and Creswick [22] in the shortrange Potts model in $2 d$.

In the end, let us mention some technical advantages of the analysis by complex- $q$ zeros compared to the complex-temperature zeros in the model with long-range interactions. The partition function of the mean-field model consisting of $N$ spins can be written as a $N$-th order polynomial in $q$, or roughly $N^{2}$-th order polynomial in temperature variable. Consequently, this allows better numerical precision of calculations in the complex- $q$ plane then those in the complex-temperature plane. For a model with power-law decaying interactions the possibilities in complex temperature plane are even more restricted. Model with $N$ spins, has $N / 2$ (for $N$ even) different parameters involving temperature, so that the partition function cannot be defined as a polynomial in some temperature variable at all.

Further investigations of the complex- $q$ zeros should be done in future in at least two directions. We plan to perform a more extended numerical calculations of the complex- $q$ zeros in the case of power-law decaying interactions. This should allow us to obtain the convergence exponent for the closest zero with more precision. In addition, it would be interesting to examine a still open question of the borderline $q_{c}(\sigma)$ dividing the first- from the second-order transition regimes within the formalism of complex- $q$ zeros. Also, on the basis of the results obtained in this paper, a possible connection between the zeros in complex- $q$ and complex-temperature plane deserves further investigation. 


\section{References}

[1] C. N. Yang and T. D. Lee, Phys. Rev. 87 (1952) 404.

[2] T. D. Lee and C. N. Yang, Phys. Rev. 87 (1952) 410.

[3] M. Fisher, in Lectures in theoretical physics vol. 12C p. 1 (University of Colorado Press, Boulder, 1965).

[4] S. Ono, Y. Karaki, M. Suzuki and C. Kawabata, J. Phys. Soc. Japan 25 (1968) 54 .

[5] S-Y. Kim and R. J. Creswick, Phys. Rev. Lett. 81 (1998) 2000.

[6] S-Y. Kim and R. J. Creswick, Physica A 281 (2000) 252.

[7] G. Bhanot, J. Stat. Phys. 60 (1990) 55.

[8] P. Martin, Potts models and related problems in statistical mechanics, World Scientific Publishing Co. Pte. Ltd., 1991, Ch. XI, and references therein.

[9] C-N. Chen, C-K. Hu and F. Y. Wu, Phys. Rev. Lett. 76 (1996) 169.

[10] S-Y. Kim, R. J. Creswick, C-N. Chen and C-K. Hu, Physica A 281 (2000) 262.

[11] P. W. Kasteleyn and C. M. Fortuin, J. Phys. Soc. Jpn. Suppl. 26 (1969) 11; C. M. Fortuin and P. W. Kasteleyn, Physica 57 (1972) 536.

[12] R. J. Baxter, J. Phys. A 19 (1986) 2821.

[13] R. J. Baxter, J. Phys. A 20 (1987) 5241.

[14] R. Shrock and S-H. Tsai, Phys. Rev. E 55 (1997) 5165.

[15] R. Shrock and S-H. Tsai, Phys. Rev. E 56 (1997) 1342.

[16] M. Roček, R. Shrock and S-H. Tsai, Physica A 259 (1998) 367.

[17] R. Shrock and S-H. Tsai, Physica A 275 (1999) 429.

[18] R. Shrock and S-H. Tsai, J. Phys. A 32 (1999) L195.

[19] A. D. Sokal, Physica A 279 (2000) 324.

[20] J. Salas and A. D. Sokal, cond-mat/0004330, to be published in J. Stat. Phys.

[21] S-C. Chang and R. Shrock, Physica A 286 (2000) 189.

[22] S-Y. Kim and R. J. Creswick, Phys. Rev. E 63 (2001) 0066107.

[23] F. Y. Wu, Rev. Mod. Phys. 54 (1982) 235.

[24] T. Kihara, Y. Midzuno and T. Shizume, J. Phys. Soc. Jpn. 9 (1954) 681. 
[25] J. P. Straley and M. E. Fisher, J. Phys. A 6 (1973) 1310.

[26] L. Mittag and J. Stephen, J. Phys. A 7 (1974) L109.

[27] R. G. Priest and T. C. Lubensky, Phys. Rev. B 13 (1976) 4159.

[28] E. Luijten and H. W. J. Blöte, Phys. Rev. Lett. 76 (1996) 1557.

[29] F. J. Dyson, Commun. Math. Phys. 12 (1969) 91.

[30] M. Aizenman, J. T. Chayes, L. Chayes and C. M. Newman, J. Stat. Phys. 50 (1988) 1.

[31] Z. Glumac and K. Uzelac, J. Phys. A 26 (1993) 5267.

[32] E. Luijten and H. W. J. Blöte, Int. J. Mod. Phys. C 6 (1995) 359.

[33] S. A. Cannas, Phys. Rev. B 52 (1995) 3034; S. A. Cannas, A. C. N. de Magalhães and F. A. Tamarit, Phys. Rev. B 61 (2000) 11521.

[34] K. Uzelac and Z. Glumac, Fizika B 6 (1997) 133.

[35] Z. Glumac and K. Uzelac, Phys. Rev. E 58 (1998) 4372.

[36] J. L. Monroe, J. Phys. A 32 (1999) 7803.

[37] E. Bayong, H. T. Diep and V. Dotsenko, Phys. Rev. Lett. 83 (1999) 14.

[38] M. Krech and E. Luijten, Phys. Rev. E 61 (2000) 2058.

[39] E. Luijten and H. Meßingfeld, Phys. Rev. Lett. 86 (2001) 5305.

[40] R. Bulirsch and J. Stoer, Numer. Math. 6 (1964) 413.

[41] M. Henkel and G. Schütz, J. Phys. A 21 (1988) 2617.

[42] J. M. Vanden Broeck and L. W. Schwartz, SIAM J. Math. Anal. 10 (1979) 658.

[43] R. Botet, R. Jullien and P. Pfeuty, Phys. Rev. Lett. 49 (1982) 478.

[44] R. Botet and R. Jullien, Phys. Rev. B 28 (1983) 3955.

[45] C. Itzykson, R.B. Pearson, and J. B. Zuber, Nucl. Phys. B 220, 415 (1983)

[46] R. J. Creswick and S-Y. Kim, Phys. Rev. E 56 (1997) 2418.

[47] K. Uzelac and Z. Glumac, Phys. Rev. Lett. 85 (2000) 5255. 


\section{Figure caption}

Fig. 1: The loci of complex- $q$ zeros of the partition function of the mean-field Potts model calculated at inverse temperatures (a) $K=K_{c}(q=0.5)=0.5$, (b) $K=K_{c}(q=2)=2$ and (c) $K=K_{t}(q=8.0)=4.540457$ for $N=25$ (open diamonds), $N=30$ (filled triangles) and $N=35$ (filled circles) spins.

Fig. 2: The locus of complex- $q$ zeros of the partition function of the mean-field Potts model calculated at inverse temperature $K=K_{M F}(q=8)=4.540457$. The full line denotes loci of zeros in the limit $N \rightarrow \infty$, obtained by the approach by the saddle point approach as a solution of the equation $\mathcal{F}_{R}=0$. Long dashed line is a circle (described in text) drawn as a guide to the eye.

Fig. 3: The complex- $q$ zeros of the partition function of the $1 d$ Potts model with power-law interactions at the $F R S$ estimate of first-order transition temperature $K=K_{t}(q=5, \sigma=0.3)=0.576$, for $N=6$ (open diamonds), $N=7$ (filled triangles), $N=8$ (open squares) and $N=9$ (filled circles) spins.

Fig. 4: The complex- $q$ zeros of the partition function of the $1 d$ Potts model with power-law interactions at the $F R S$ estimate of second-order transition temperature $K=K_{c}(q=2, \sigma=0.8)=0.8230$, for $N=6$ (open diamonds), $N=7$ (filled triangles), $N=8$ (open squares) and $N=9$ (filled circles) spins. 
Fig. 1 (a)

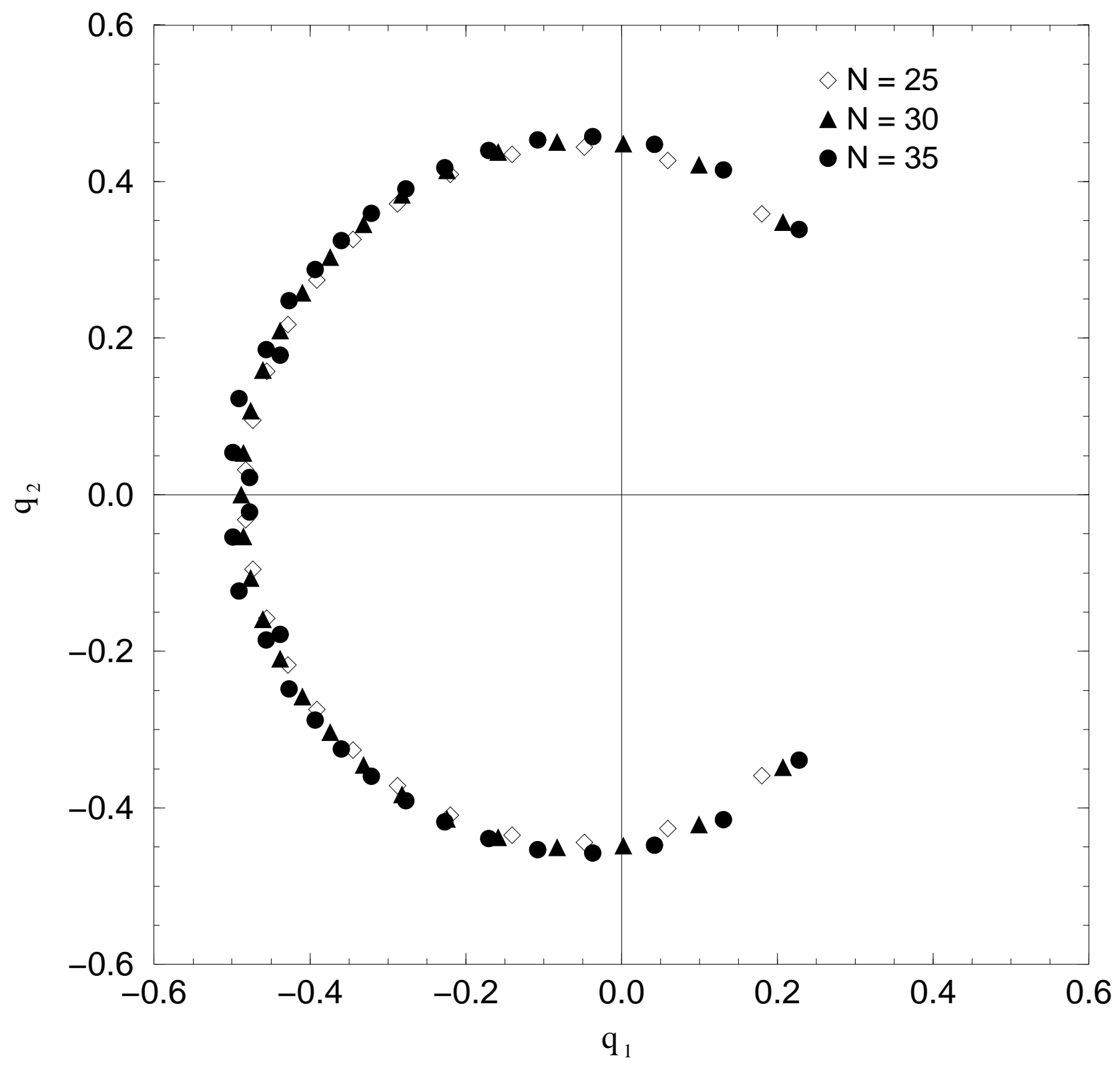


Fig. 1 (b)

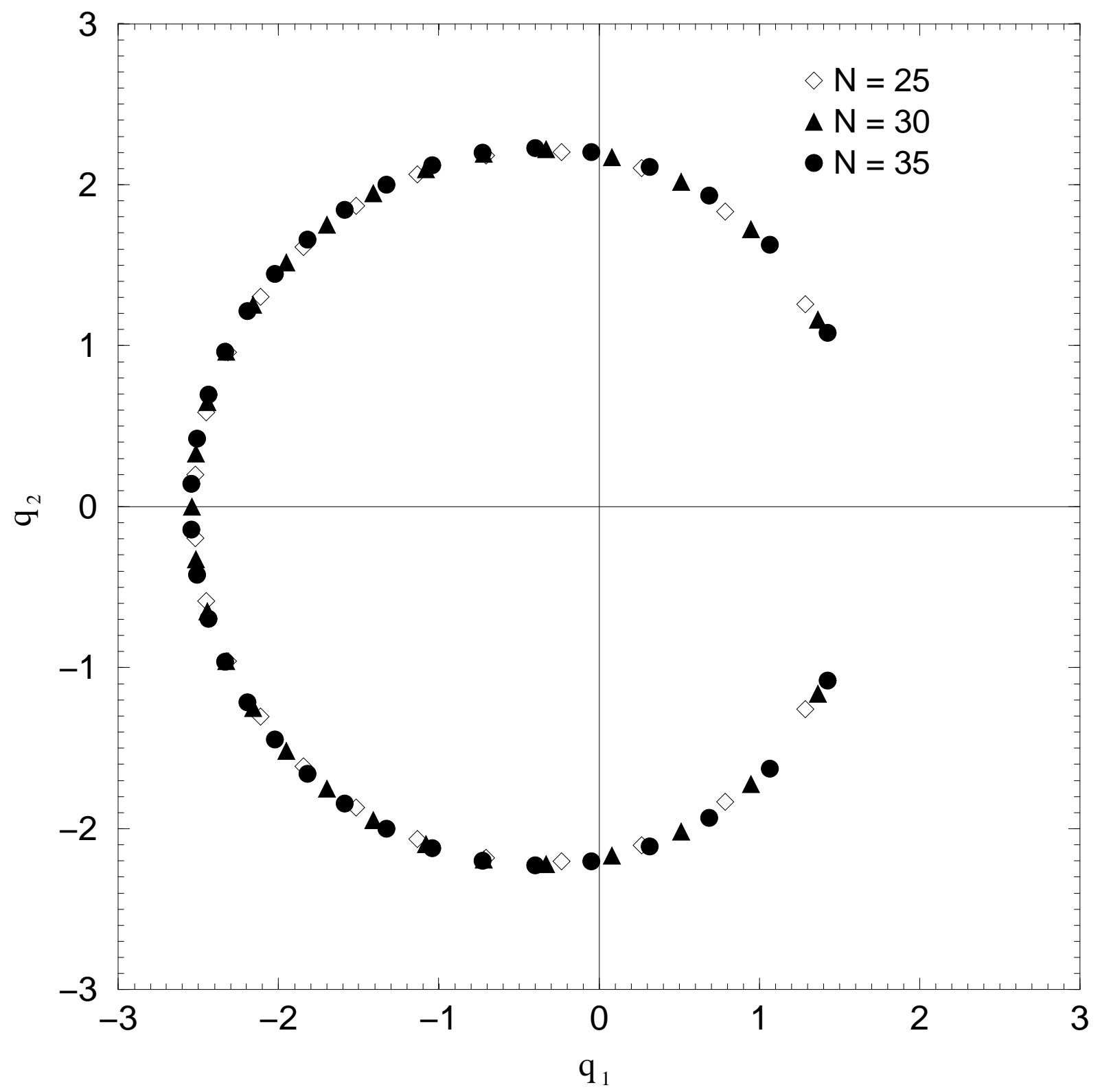


Fig. 1 (c)

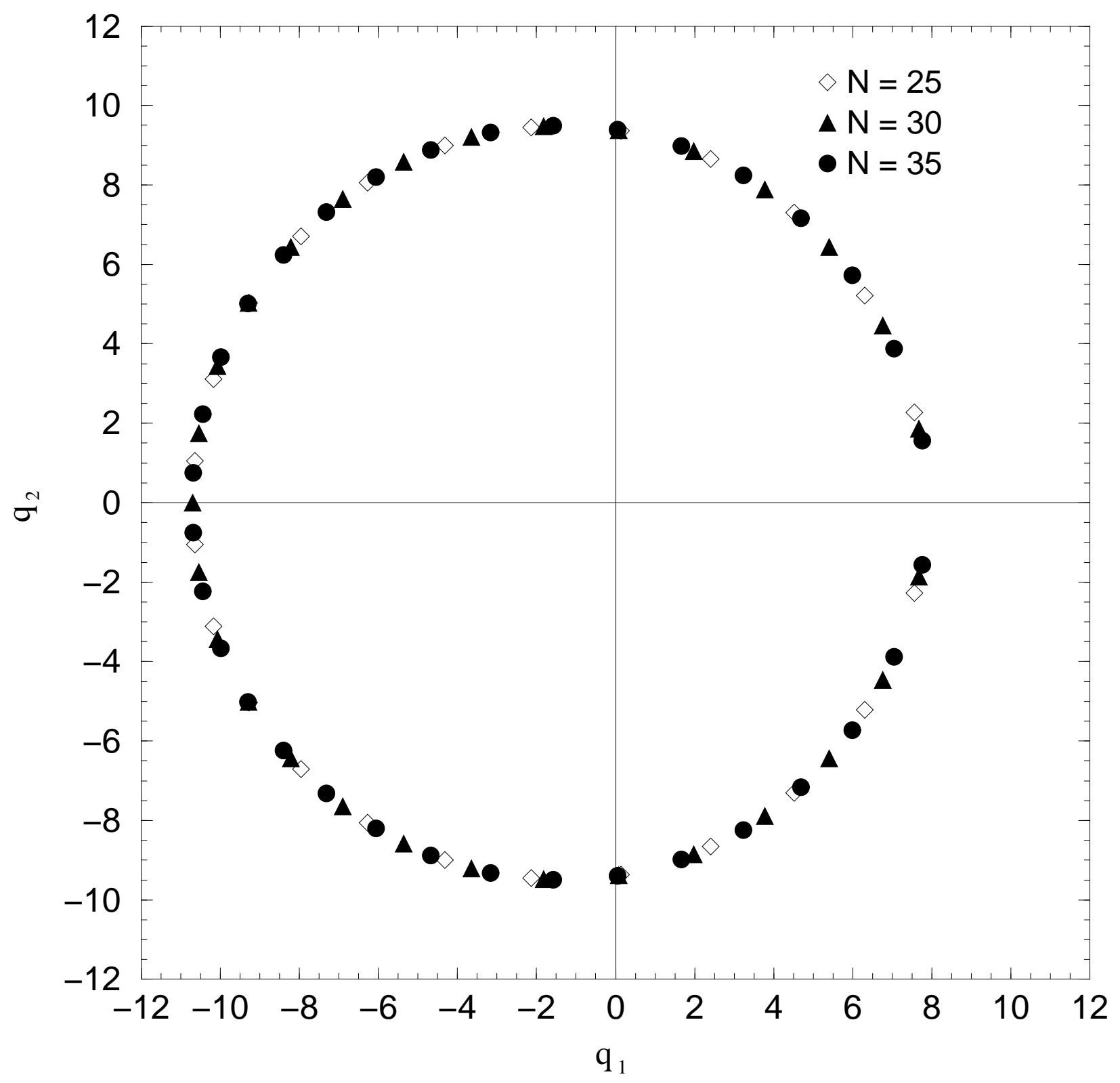


Fig. 2

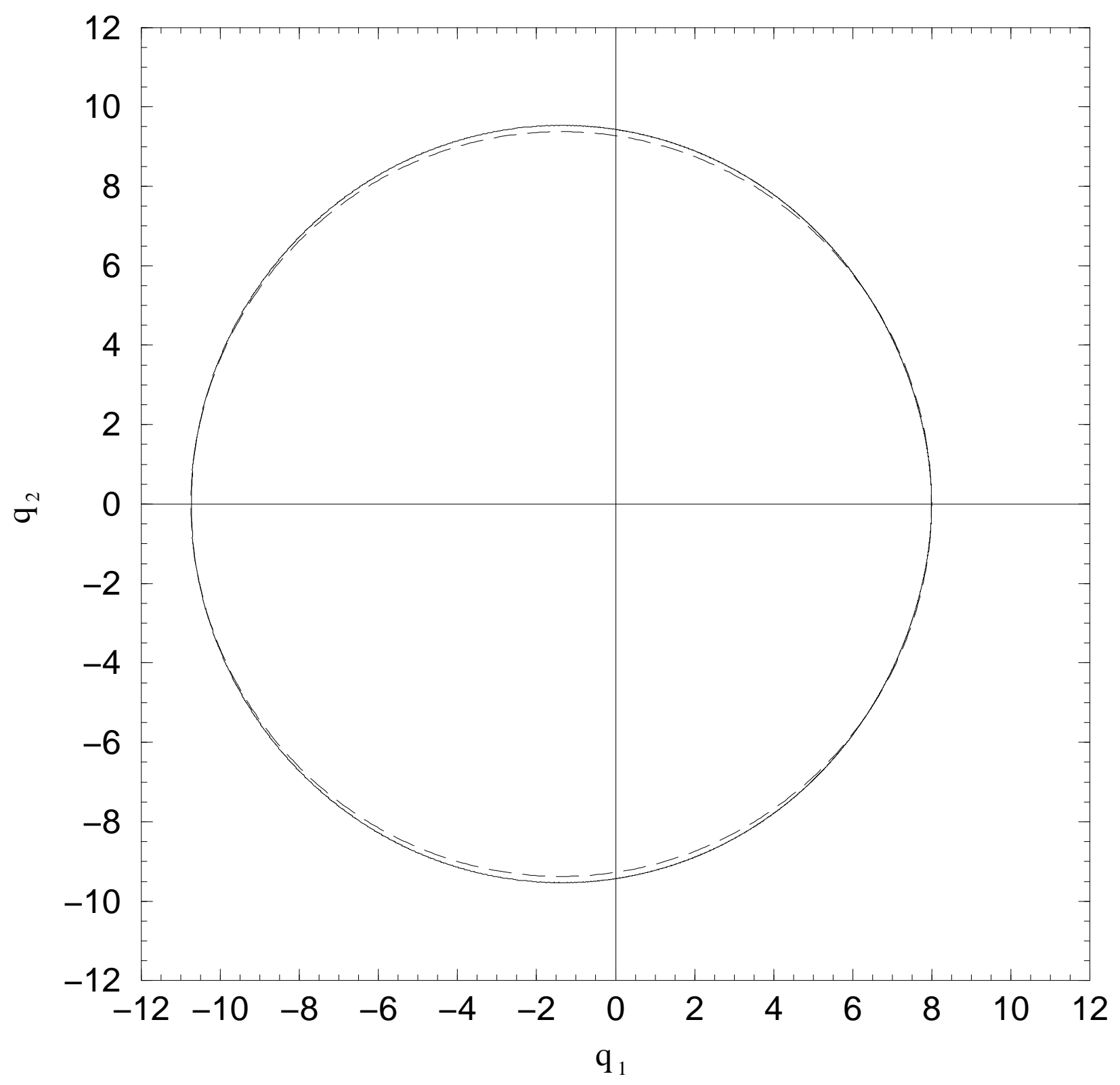


Fig. 3

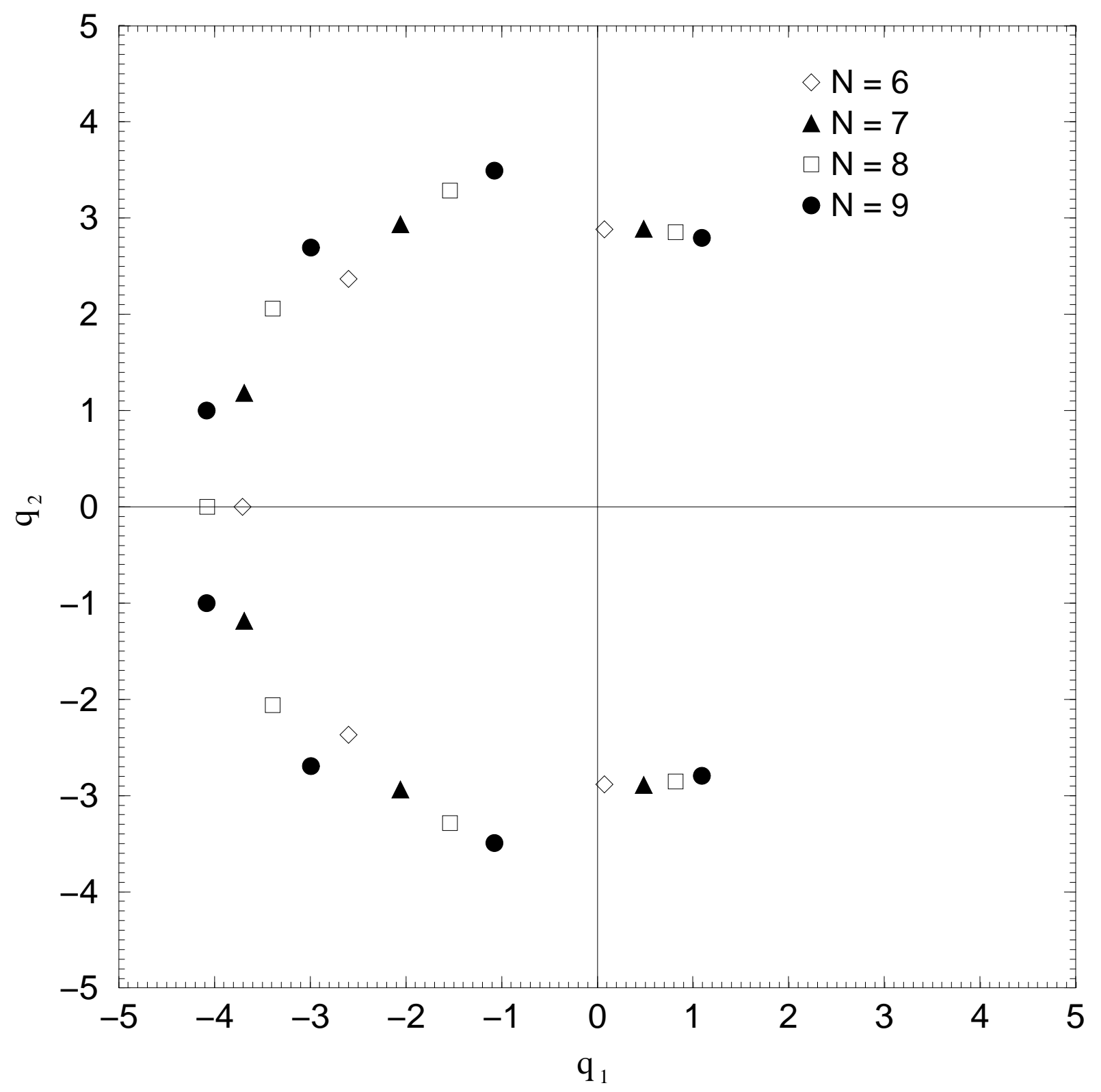


Fig. 4

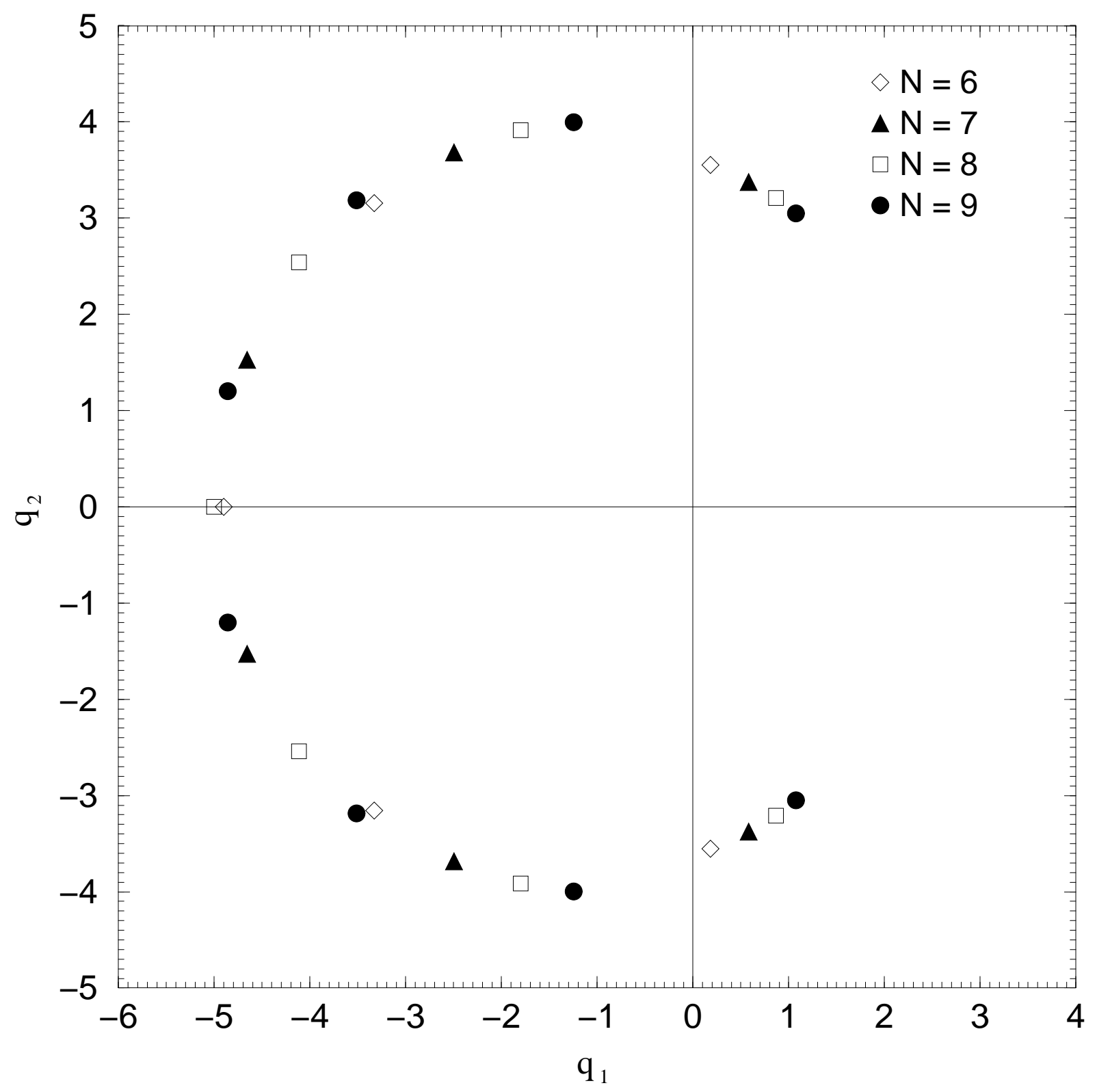

